\title{
QUALITATIVE RESEARCH ON HUNGARIAN MOTHERS' SOCIAL SITUATION AND MENTAL HEALTH DURING THE TIME OF THE COVID-19 PANDEMIC
}

\author{
RÉKa Geambașu, ${ }^{1}$ Orsolya Gergely, ${ }^{2}$ BeÁta NaGY, ${ }^{3}$ Nikolett Somogy ${ }^{4}$
}

Due to the social distancing measures ordered as protection against mass infection during the 2020 COVID-19 outbreak, most Hungarian families were confined to home quarantine. Schools, kindergartens, and nursery schools were closed between 16 March and early June. Because grandparents belonged to one of the most vulnerable groups, families were asked not to involve them in childcare until the end of the pandemic in order to avoid their infection by younger family members. Companies switched to using the home office when possible, and the government asked the population not to leave their homes except for essential reasons. As a result, many parents worked from home and provided care for their children at the same time.

In families with school-aged children, facilitating home-schooling was also a task entrusted to parents, adding one more burden to their already busy days. Parents could not rely on their usual childcare methods, thus they needed to tend to home-schooling and work-related obligations within a limited timeframe. As all these changes happened overnight, and without a carefully planned transition to digital teaching methods, both teachers and parents were stressed by the new requirements. Given the deep gender segregation in the labor market and the traditional gender regime, women bore the majority of the burden in these areas. Being in full-time employment and doing the "second shift" (Hochschild, 2012) has been a familiar experience for Hungarian working women for several decades. However, it was a novel experience to be required to fulfil these sometimes conflicting responsibilities at the same time and in the same place.

\footnotetext{
1 Babeș-Bolyai University, Cluj/Kolozsvár, reka.geambasu@ubbcluj.ro (corresponding author)

2 Sapientia - Hungarian University of Transylvania, Miercurea Ciuc/Csíkszereda

3 Corvinus University of Budapest

4 University of Antwerp
} 
In our research project ${ }^{5}$ we were interested in examining what toll this took on the social situation and mental health of working women; moreover, what strategies and tools they used to survive this period. We expected the high level of work-family conflict to be explained by time scarcity (due to the zero-sum nature of time); by the demands-and-resources approach (work demands deplete resources, which has an effect on private life, and vice versa); or by role incompatibility (Greenhaus \& Beutell, 1985). Since work was essentially done at home, there was an elevated risk of the blurring of work-family roles as well. Based on earlier literature, it is known that the level of work-family conflict experienced by women and men has been converging. The reason for the closing gap may be that women find more effective ways of coping with the related stress, and that men exhibit more intensive fathering behavior, thus experiencing similar stress to women. Nevertheless, women and men typically choose different coping strategies (Geszler, 2020).

Based on earlier research findings and recent international reports, we formulated two different assumptions about the changes taking place in Hungarian dual-earner families who were raising small children in spring 2020. On the one hand, as women/mothers/caregivers were now at home and accessible full-time, the further re-traditionalization of norms and expectations may have occurred. On the other hand, as men/fathers/caretakers/ main breadwinners were also at home full-time, and were more accessible than previously for family-related responsibilities, some de-traditionalization may have occurred (men could "help" more with children, housework, and elderly care). It was thus an exciting question how these patterns had changed, with special attention paid to mothers' lives and experiences. Based on the statements discussed above, the research was thus designed to explore changes in mothers' lives due to working using a home office, home schooling, and an intense family life, as perceived by Hungarian interviewees.

\footnotetext{
5 More information about the research is available on ResearchGate (https://www.researchgate. net/project/Mothers-during-the-COVID-19-quarantine) and on Zenodo.org (https://zenodo.org/ communities/mothers-huro-covid19/?page $=1 \&$ size=20\&amp;size $=20$ ).
} 


\section{THE QUALITATIVE RESEARCH FOCUSED ON THE FOLLOWING FOUR QUESTIONS}

\section{How do mothers (families) usually cope with the dual and parallel burden of work and childcare?}

This involves the investigation of the challenges and difficulties women face connected to childcare and child-related tasks (past and present). We expected that mothers would seek to rise to the expectation of being good/perfect, intensive mothers. Thus, through "exploiting themselves" they would put even more effort into meeting these norms, relegating themselves and their own needs to the background.

2. How does the situation in which parents work from home and provide childcare affect the household division of work between women and men?

Here we focused on the performance of unpaid labor at home (past and present), with particular attention to changes in routines and their explanations. The ideologies behind the division of labor at home, and satisfaction with this situation, were crucial elements of the investigation. Because of the traditional gender regime at home, we expected that women would be completing the majority of these tasks, but we also they projected that they would report that fathers "helped" to reduce tension.

3. How do working mothers experience and handle work-family conflict in this situation?

Mothers' and fathers' previous and present work experience and work-life balance were the focus of this question. Mothers were expected to report attempting to resolve related conflicts by working more extensively, and always being online. We anticipated the expansion of mothers' working days, thus their permanent exhaustion.

\section{How does this situation affect the mental health of mothers?}

In our investigation of mothers' mental status, we were interested in exploring the presence of both negative and positive social, economic, and natural environmental factors during the pandemic. Anxiety connected to job loss, children's suboptimal academic progress, or perceptions of poor mothering could be accelerated by peer pressure disseminated on social media. 


\section{SAMPLE}

We interviewed 52 Hungarian mothers who were residents of Hungary and Romania (with one exception, all Romanian interviewees were from Transylvania, and all of them were of Hungarian ethnicity), who were living in dual-earner households, were married or cohabitating, and were raising at least one child under the age of 15 . Interviewees were recruited through social media ads, several dedicated on-line groups, and through the researchers' personal networks. In order to tackle the homogeneity of the sample, we also applied the snowball technique as a means of contacting additional interviewees. Regarding the layers of the sample, the fact that the mother's job was required to be carried out from home created a restriction. We aimed at reaching women in highflexibility, independent, and creative jobs, related to which they had the everyday experience of working online, as well as other women who were undertaking more routine administrative work with limited flexibility. We also tried to avoid geographical bias and to interview women living in Budapest and in bigger cities and villages, as all semi-structured interviews were carried out via online platforms or phone. Interviews lasted around one hour, and all were recorded and typed up. Texts were analyzed with NVivo software. The Transylvanian sample is dominated by schoolteachers, which gave us an additional opportunity to analyze the special status of teachers during the pandemic.

\section{PRELIMINARY FINDINGS}

Mothers perceived the time spent in quarantine at home as an opportunity to be even more present in their children's lives. Home schooling, however, multiplied the burden on mothers with regard to ensuring the adequate progress of children in school.

The gender inequality in invisible and unpaid domestic work was not caused by COVID-19, but the epidemic-induced quarantine reinforced and deepened preexisting gender inequalities.

Our interviewees' lives had changed radically: the priority of mothers became caring for their children and supervising their school-related activity. In comparison, the lives of their spouses/partners changed much less.

Social distancing, working from home, permanent childcare, and homeschooling-related duties put women under significant pressure from several directions, negatively affecting their mental health. 


\section{REFERENCES}

Geszler, Nikolett (2020) Agency and capabilities in managerial positions: Hungarian fathers' use of workplace flexibility. Social Inclusion, Vol. 8, No. 4, pp. 61-71, DOI: http://dx.doi.org/10.17645/si.v8i4.2969

Greenhaus, Jeffrey. H. - Nicholas J. Beutell (1985) Sources of conflict between work and family roles. Academy of Management Review, Vol. 10. No. 1, pp. 76-88, DOI: $10.2307 / 258214$

Hochschild, Arlie (2012) The Outsourced Self: Intimate Life in Market Times. New York, Metropolitan Press 
